TRANSACTIONS OF THE

AMERICAN MATHEMATICAL SOCIETY

Volume 354, Number 5 , Pages 1861-1868

S 0002-9947(02)02879-9

Article electronically published on January 8, 2002

\title{
ON CREPANT RESOLUTION OF SOME HYPERSURFACE SINGULARITIES AND A CRITERION FOR UFD
}

\author{
HUI-WEN LIN
}

\begin{abstract}
In this article, we find some diagonal hypersurfaces that admit crepant resolutions. We also give a criterion for unique factorization domains.
\end{abstract}

\section{INTRODUCTION}

Let $X$ be a normal complex algebraic variety which is $\mathbf{Q}$-Gorenstein, namely, a (non-zero) integer multiple of the canonical divisor $K_{X}$ of $X$ gives rise to a line bundle on $X$. We say that $X$ has a projective crepant resolution if there exists a projective birational morphism $\phi: Y \rightarrow X$ such that $Y$ is smooth and $K_{Y}=\phi^{*} K_{X}$. This is a special type of canonical singularity, studied in minimal model theory, which also plays an important role in recent research in the theory of Calabi-Yau manifolds.

In the two dimensional case, singularities admitting projective crepant resolutions are exactly canonical singularities, and have been studied extensively for a long time. They are isolated quotient singularities $X=\mathbf{C}^{2} / G$ with $G \subset \operatorname{SL}(2, \mathbf{C})$ a finite subgroup (so-called Kleinian singularities). They can also be represented as hypersurfaces in $\mathbf{C}^{3}$ through explicit A-D-E equations:

$$
\begin{array}{ll}
A_{n}: & X^{2}+Y^{2}+Z^{n+1}=0, \quad n \geq 1 ; \\
D_{n}: & X^{2}+Y^{2} Z+Z^{n-1}=0, \quad n \geq 4 ; \\
E_{6}: & X^{2}+Y^{3}+Z^{4}=0 ; \\
E_{7}: & X^{2}+Y^{3}+Y Z^{3}=0 ; \\
E_{8}: & X^{2}+Y^{3}+Z^{5}=0 .
\end{array}
$$

In the higher dimensional cases, the above two descriptions give rise to two different types of generalization. It has been proved recently by Roan $\left[\mathrm{RO}\right.$ that $\mathbf{C}^{3} / G$ with $G$ a finite subgroup of $\mathrm{SL}(3, \mathbf{C})$ always admits projective crepant resolutions. However, it is a consequence of Schlessinger's theorem $[\mathrm{Sc}$ that if $n \geq 3$ then an isolated quotient singularity $\mathbf{C}^{n} / G(G$ a finite subgroup of $\mathrm{SL}(n, \mathbf{C}))$ is not smoothable, hence can never be a hypersurface singularity. Alternatively, this follows from the fact that such a quotient singularity $\mathbf{C}^{n} / G$ is a hypersurface singularity if and only if the singular locus is of codimension 2 (Kac-Watanabe [KaW]), so the singularity is never isolated.

Received by the editors January 10, 2000 and, in revised form, March 22, 2001.

2000 Mathematics Subject Classification. Primary 14E15, 13A99.

Key words and phrases. Crepant resolution, UFD.

Supported by a CTS post-doctor-fellowship. 
In fact, in dimension three, the structure of canonical singularities has been well understood since the 80's, through work of Reid, Kawamata and Kollár. We surmarize some of their results in the following statement:

Theorem 1. Let $X$ be a three dimensional algebraic variety with at most canonical singularities. Then

1. "Terminalization" [Re2, Main Theorem]: there exists a projective crepant partial resolution $Y \rightarrow X$ such that $Y$ has at most terminal sigularities. Moreover,

2. "Q-factorialization" [Ka, Corollary 5.4]: Y can be choosen to be $\mathbf{Q}$-factorial, possibly by a further projective small resolution.

3. "Quasi-uniqueness" [Ko Corollary 4.11]: Y may not be unique, but any two such $Y$ 's admit the same finite set of germs of isolated singularities.

In this note we consider hypersurfaces $X \subset \mathbf{C}^{n+1}$ with an isolated singularity $0 \in X$ defined by

$$
f\left(x_{0}, \ldots, x_{n}\right):=x_{0}^{a_{0}}+\cdots+x_{n}^{a_{n}}=0,
$$

where $a_{i} \geq 2$. Our goal is to find examples of $a_{i}$ 's so that $X$ admits crepant resolutions. To get a better idea of the problem, we recall another theorem of Reid [Re1, Proposition 4.3] which is valid for arbitrary dimension $n$ :

Theorem 2. Let $X$ be the hypersurface given by (1.1). Then

1. $X$ has at most canonical singularities if and only if $\sum \frac{1}{a_{i}}>1$. And

2. $X$ has at most terminal singularities if and only if $\sum \frac{1}{a_{i}}>1+\frac{1}{\text { l.c.m. }\left\{a_{i}\right\}}$.

In dimension three, our strategy goes in the following way. By Theorem 1, one should first find the crepant partial resolution $\phi: Y \rightarrow X$. This step is not hard to do directly, we simply follow the method of Reid [Re1] via toric (weighted) blow-ups (see $\S \S 3,4)$. Then we use a monodromy technique of Milnor Mi to test the analytical $\mathbf{Q}$-factoriality of $Y$ (see $\S 2$ ). Since analytical factoriality implies factoriality, this may also be used for algebraic purposes.

In good cases the $Y$ constructed is already $\mathbf{Q}$-factorial; then Theorem 1.3 shows that $X$ has crepant resolutions only if $Y$ is smooth. In the bad case, namely if the obtained $Y$ is not $\mathbf{Q}$-factorial, then we need to perform the small resolution in Theorem 1.2. This step is much harder and will not be considered in this note, but we wish to come back to it in a later work.

We summarize our results in the following:

Theorem A. Let $X=\left\{x \in \mathbf{C}^{4} \mid f(x)=0\right\}$ be a three dimensional hypersurface. Then:

1. For $f=x_{0}^{3}+x_{1}^{3}+x_{2}^{3}+x_{3}^{k}, X$ has a projective crepant resolution if and only if $k \equiv 0,1(\bmod 3)$.

2. For $f=x_{0}^{2}+x_{1}^{4}+x_{2}^{4}+x_{3}^{k}$, X has a projective crepant resolution if $k \equiv 0,1$ $(\bmod 4)$, and $X$ has no projective crepant resolution if $k \equiv-1(\bmod 4)$.

3. For $f=x_{0}^{2}+x_{1}^{3}+x_{2}^{6}+x_{3}^{k}, X$ has a projective crepant resolution if $k \equiv 0,1$ $(\bmod 6)$, and $X$ has no projective crepant resolution if $k \equiv-1(\bmod 6)$.

For higher dimensional cases $X=\left\{x \in \mathbf{C}^{n+1} \mid f(x)=0\right\}$, we have 
4. For $f=x_{0}^{n}+x_{1}^{n}+\cdots+x_{n-1}^{n}+x_{n}^{k}$, X has a projective crepant resolution if $k \equiv 0,1(\bmod n)$, and $X$ has no projective crepant resolution if $1<k<n$.

Theorem B. Let $f=x_{0}^{a_{0}}+x_{1}^{a_{1}}+x_{2}^{a_{2}}+x_{3}^{a_{3}}, A=\mathbf{C}\left[x_{0}, \ldots, x_{3}\right] /(f)$ and $\hat{A}=$ $\mathbf{C}\left[\left[x_{0}, \ldots, x_{3}\right]\right] /(f)$. Then $A$ is an UFD if and only if $\hat{A}$ is an UFD. Moreover, if $\sum \frac{1}{a_{i}}>1$ (that is, the hypersurface $f=0$ has only canonical singularities), then to be an UFD is equivalent to the non-existence of integers $n_{i}, 1 \leq n_{i} \leq a_{i}-1$, $i=0, \ldots, 3$ such that

$$
\frac{n_{0}}{a_{0}}+\frac{n_{1}}{a_{1}}+\frac{n_{2}}{a_{2}}+\frac{n_{3}}{a_{3}}=2 .
$$

The first statement in Theorem B is due to Flenner [Fl, (4.5) Satz]. But our criterion (1.2) seems to be new. We would like to remark that Theorem B holds true for dimension $\geq 4$ (with the same $f$ ); however, this is a simple consequence of Grothendieck's result (see Proposition 3.2).

The author would like to thank C.-L. Wang for pointing out to her the relation between factoriality and the monodromy argument in Milnor's book [Mi]. She also wants to give special thanks to the referee, who provided valuable suggestions to make the paper more clear and organized.

\section{Monodromy AND FACtORIALity}

In this section we will prove Theorem $\mathrm{B}$, which in turn will be used to prove Theorem A in later sections.

A normal algebraic variety $X$ is called factorial (resp. analytically factorial) if all its local rings (resp. completion of local rings) are factorial, that is, UFD's. Geometrically $X$ being factorial means that any Weil divisor of $X$ is Cartier. In general, analytical factoriality implies factoriality (due to Mori, see e.g. [Fl, (1.2) Lemma]), but not the converse (see for example [Sa, p.41 Corollary 2]). Also we say that $X$ is $\mathbf{Q}$-factorial if every Weil divisor of $X$ is a $\mathbf{Q}$-Cartier divisor. When $(0 \in X)$ is a germ of an isolated singular point, $X$ is analytically factorial (resp. Q-factorial) if and only if $\operatorname{Pic}(X-0)$ is zero (resp. torsion).

Let $X=\left\{x \in \mathbf{C}^{n+1} \mid f(x)=0\right\}(n \geq 3)$ be a hypersurface with an isolated singular point at 0 , and $S$ a sphere of small radius centered at 0 . Then $K:=S \cap X$ is a differentiable manifold of real dimension $2 n-1$. In [Mi], Milnor constructed a fibration over the unit circle $S^{1}$ (we follow closely the notation there)

$$
\Phi: S-K \longrightarrow S^{1}, \quad x \mapsto \frac{f(x)}{|f(x)|} \in S^{1}
$$

such that $K$ is the common boundary of each fiber $F_{\theta}:=\Phi^{-1}(\theta)$. Let $F$ be a fixed reference fiber and $h: F \rightarrow F$ the monodromy mapping around the circle. Milnor showed that $K$ is $(n-2)$-connected and $F$ has the homotopy type of $n$-dimensional spheres with one point in each sphere identified together. Hence $H_{i}(F ; \mathbf{Z})$ is nontrivial only when $i=0$ or $n$ and $H_{n}(F ; \mathbf{Z})$ is a free abelian group. He then showed the following exact sequence:

$$
H_{n}(F ; \mathbf{Z}) \stackrel{I-h_{*}}{\longrightarrow} H_{n}(F ; \mathbf{Z}) \longrightarrow H_{n}(S-K ; \mathbf{Z}) \longrightarrow 0 .
$$

With these, we may deduce (cf. [Mi, Theorem 8.5]):

Proposition 3. Assume that the hypersurface germ $(0 \in X)$ has only rational singularities and has dimension $n \geq 3$. Then 
1. Every $\mathbf{Q}$-Cartier divisor is automatically Cartier, and hence that $\mathbf{Q}$-factoriality is equivalent to factoriality.

2. (Grothendieck) If $n \geq 4$ then $X$ is analytically factorial at 0 .

3. If $n=3, X$ is analytically factorial if and only if $\operatorname{det}\left(I-h_{*}\right) \neq 0$ on $H_{3}(F ; \mathbf{Z})$.

Proof. For isolated rational singularities, Flenner [Fl, (6.1) Satz] has shown that

$$
\operatorname{Pic}(X-0) \cong H^{2}(K ; \mathbf{Z}) \text {. }
$$

Since $K$ is simply connected, the universal coefficient theorem shows that $H^{2}(K ; \mathbf{Z})$ $\cong H_{2}(K ; \mathbf{Z})^{*}$ has no torsions. If $D$ is a Weil divisor, then it defines an element $[D]$ in the local divisor class group $\operatorname{Pic}(X-0)$, and $D$ is Cartier if and only if $[D]=0$ in $\operatorname{Pic}(X-0)$ since $X$ is a contractible neighborhood of 0 . Now if $\ell D$ is Cartier, then $[\ell D]=0$ in $\operatorname{Pic}(X-0)$. This implies that $[D]$ is torsion; hence by $(2.3)[D]=0$. That is, $D$ is Cartier.

If $n \geq 4$, then $K$ is $(n-2) \geq 2$ connected; hence $H^{2}(K ; \mathbf{Z})=0$ and $X$ is analytically factorial at 0 .

For $n=3$, by Poincaré duality $H_{2}(K ; \mathbf{Z}) \cong H^{3}(K ; \mathbf{Z})$ and by Alexander duality $H^{3}(K ; \mathbf{Z}) \cong H_{3}(S-K ; \mathbf{Z})$. Since torsions will be cancelled out after taking dual, we find that $X$ is factorial if and only if $H_{3}(S-K ; \mathbf{Z})$ is a torsion group. By (2.2), this is equivalent to $I-h_{*}$ inducing an isomorphism of $\mathbf{Q}$-vector spaces. That is, $\operatorname{det}\left(I-h_{*}\right) \neq 0$.

Q.E.D.

Proof of Theorem B. The statement that $A$ is an UFD if and only if $\hat{A}$ is an UFD is due to Flenner, so we will only prove the criterion (1.2). In case $f$ is given by (1.1), the formula for $\operatorname{det}\left(I-h_{*}\right)$ has been determined by Brieskorn and Pham (see [Mi, Theorem 9.1]):

$$
\operatorname{det}\left(I-h_{*}\right)=\prod_{w_{i}^{a_{i}}=1, w_{i} \neq 1}\left(1-w_{0} \cdots w_{3}\right) .
$$

A result due to Elkik and Flenner (see e.g. [Re3, $[\overline{\mathrm{KMM}}$ ) says that canonical singularities are all rational. So by assumption on $f, X=\operatorname{Spec}(A)$ is rational. Proposition 3.3 and (2.4) then imply that $\hat{A}$ is an UFD if and only if

$$
w_{0} \cdots w_{3} \neq 1, \quad \forall w_{i} \neq 1, w_{i}^{a_{i}}=1 .
$$

Write $w_{i}=e^{2 \pi \sqrt{-1} n_{i} / a_{i}}$; then it is clear that (2.5) is equivalent to (1.2), because

$$
\left(\frac{n_{0}}{a_{0}}+\frac{n_{1}}{a_{1}}+\frac{n_{2}}{a_{2}}+\frac{n_{3}}{a_{3}}\right)+\left(\frac{a_{0}-n_{0}}{a_{0}}+\frac{a_{1}-n_{1}}{a_{1}}+\frac{a_{2}-n_{2}}{a_{2}}+\frac{a_{3}-n_{3}}{a_{3}}\right)=4
$$

and each term in the LHS is an integer bigger than 1.

Q.E.D.

Remark 4. In fact, rational Gorenstein singularities are exactly canonical singularities of index one ([Re3], [KMM] $)$.

\section{Standard Crepant Blow Up}

In this section we prove part 1 and part 4 of the Main Theorem: let $f=x_{0}^{n}+$ $x_{1}^{n}+\cdots+x_{n-1}^{n}+x_{n}^{k}$ and $X=\left\{x \in \mathbf{C}^{n+1} \mid f(x)=0\right\}$ with $k \geq n$. In general, if mult $_{0}(X)=m$ then for $\phi: \tilde{\mathbf{C}}^{n+1} \rightarrow \mathbf{C}^{n+1}$ the blow-up at 0 with exceptional divisor $E$ we have

$$
K_{\tilde{\mathbf{C}}^{n+1}}=\phi^{*} K_{\mathbf{C}^{n+1}}+n E,
$$


and if $X^{\prime}$ is the proper transform of $X$ in $\tilde{\mathbf{C}}^{n+1}$ then, as pull-back of Cartier divisors,

$$
\phi^{*} X=X^{\prime}+m E .
$$

Since $\tilde{\mathbf{C}}^{n+1}$ is smooth, $X^{\prime}$ is Gorenstein and we may apply the adjunction formula

$$
K_{X^{\prime}}=\left.\left(K_{\tilde{\mathbf{C}}^{n+1}}+X^{\prime}\right)\right|_{X^{\prime}} \text { and } K_{X}=\left.\left(K_{\mathbf{C}^{n+1}}+X\right)\right|_{X} .
$$

Putting these together, we get the canonical bundle formula for a single blow-up:

$$
K_{X^{\prime}}=\phi^{*} K_{X}+\left.(n-m) E\right|_{X^{\prime}} .
$$

In our case $m=n$, so $\phi: X^{\prime} \rightarrow X$ is a crepant blow-up.

Now let $\tilde{\mathbf{C}}^{n+1}=\bigcup_{i=0}^{n} U_{i}$ with $U_{i} \cong \mathbf{C}^{n+1}$ affine charts, and let $k \geq n$.

Claim 5. $X^{\prime}$ is nonsingular on $U_{i}$ for $i \neq n$. On $U_{n}, X^{\prime}$ is a hypersurface with equation $g=y_{0}^{n}+y_{1}^{n}+\cdots+y_{n-1}^{n}+y_{n}^{k-n}=0$.

In fact, consider $U_{0}$ with coordinates $\left(y_{0}, \ldots, y_{n}\right)$; then the morphism $\phi$ is given by $x_{0}=y_{0}, x_{1}=y_{0} y_{1}, \ldots, x_{n}=y_{0} y_{n}$. So

$$
\begin{aligned}
\phi^{*} f(y) & =f(\phi(y))=f\left(y_{0}, y_{0} y_{1}, \ldots, y_{0} y_{n}\right) \\
& =y_{0}^{n}+y_{0}^{n} y_{1}^{n}+\cdots+y_{0}^{n} y_{n-1}^{n}+y_{0}^{k} y_{n}^{k} \\
& =y_{0}^{n}\left(1+y_{1}^{n}+\cdots+y_{n-1}^{n}+y_{0}^{k-n} y_{n}^{k}\right)=y_{0}^{n} g(y) .
\end{aligned}
$$

The singular set is on the exceptional divisor $E \cap X^{\prime}$. On $U_{0}$ it is given by $y_{0}=0$ and $g(y)=0$. The singular set satisfies $D g=0$, that is,

$$
D g=\left((k-n) y_{0}^{k-n-1} y_{n}^{k}, n y_{1}^{n-1}, \ldots, n y_{n-1}^{n-1}, k y_{0}^{k-n} y_{n}^{k-1}\right)=0 .
$$

This forces $y_{1}=\cdots=y_{n-1}=0$ and $y_{0} y_{n}=0$. On such a set, $g(y)=1 \neq 0$, hence $X^{\prime}$ is nonsingular on it. Similarly $X^{\prime}$ is nonsingular on $U_{i}$ for $i \neq n$.

Now consider $U_{n}$ with coordinates $\left(y_{0}, \ldots, y_{n}\right)$. The morphism $\phi$ is now given by $x_{0}=y_{n} y_{0}, \ldots, x_{n-1}=y_{n} y_{n-1}, x_{n}=y_{n}$. So

$$
\begin{aligned}
\phi^{*} f(y) & =f(\phi(y))=f\left(y_{n} y_{0}, \ldots, y_{n} y_{n-1}, y_{n}\right) \\
& =y_{n}^{n} y_{0}^{n}+\cdots+y_{n}^{n} y_{n-1}^{n}+y_{n}^{k} \\
& =y_{n}^{n}\left(y_{0}^{n}+\cdots+y_{n-1}^{n}+y_{n}^{k-n}\right)=y_{n}^{n} g(y) .
\end{aligned}
$$

The claim is thus proved.

For the theorem, if $k \equiv 0,1 \bmod n$, then after a finite number of crepant blowups we arrive at the case $k=0$ or $k=1$. In both cases we get smooth varieties, and hence we get the crepant resolution of $X$. For other cases, after a finite number of crepant blow-ups we arrive at a hypersurface $X^{\prime}$ when $1<k<n$. These are terminal singularities since $\sum \frac{1}{a_{i}}=n \cdot \frac{1}{n}+\frac{1}{k}>1+\frac{1}{n}$.

Consider first the case $n=3$. Then $k=2$, and there are no positive integer solutions of

$$
\frac{1}{2}+\frac{a}{3}+\frac{b}{3}+\frac{c}{3}=2
$$

By Theorem B, $X^{\prime}$ is factorial but singular. Hence by Theorem $1.3, X$ has no crepant resolution.

Now assume that $n \geq 4$ and $1<k<n$. Then $X^{\prime}$ is factorial by Proposition 3.2. If there is a further projective small resolution $\psi: Y \rightarrow X^{\prime}$, then there is a nontrivial divisor $D$ on $Y$ with $D . C>0$ for some curve $C$ lying on a fiber of $\psi$. But since $\psi$ is an isomorphism in codimension one, $D=\psi^{*} L$ for some Cartier divisor $L$ in $X^{\prime}$. But then D.C $=\psi^{*} L . C=L . \psi(C)=0$, a contradiction.

Q.E.D. 
Remark 6 . This later argument only works for $1<k<n$, because of the nonuniqueness of "Terminalization" and lack of a theorem like Kollár's result in higher dimensions.

\section{Weighted Crepant Blow-Up}

In this section we prove part 2 and part 3 of the Main Theorem. Namely, we consider the equation $f=x_{0}^{2}+x_{1}^{4}+x_{2}^{4}+x_{3}^{k}$ or $f=x_{0}^{2}+x_{1}^{3}+x_{2}^{6}+x_{3}^{k}$. Since these are double points, the standard blow-up at 0 is not a crepant blow-up. In fact it will even create more complicated singularities (one dimensional or even non-normal).

In Reid's process of terminalization [Re1, he introduced two more weighted blowups to resolve the problem. Namely, he showed that for index one isolated canonical singularities, one may achieve the terminal partial resolution by only three types of (weighted) blow-ups: their weights are $(1,1,1,1),(2,1,1,1)$ and $(3,2,1,1)$. The two equations we take here are the simplest ones that require weighted blow-ups.

Let $X=\left\{x \in \mathbf{C}^{n+1} \mid f(x)=0\right\}$. For a general weight $\alpha$, let $\phi: \tilde{\mathbf{C}}^{n+1} \rightarrow \mathbf{C}^{n+1}$ be the $\alpha$-blow up at the point 0 , and $E$ its exceptional divisor. $E$ is isomorphic to the $\alpha$-weighted projective space. We have the following well known computation via toric geometry (cf. [Re3]):

$$
K_{\tilde{\mathbf{C}}^{n+1}}=\phi^{*} K_{\mathbf{C}^{n+1}}+\left(\alpha\left(x_{0} x_{1} \cdots x_{n}\right)-1\right) E,
$$

and if $X^{\prime}$ is the proper transform of $X$ in $\tilde{\mathbf{C}}^{n+1}$, then, as pull-back of Cartier divisors,

$$
\phi^{*} X=X^{\prime}+\alpha(f) E
$$

here $\alpha(f):=\min \left\{\alpha\left(x^{u}\right) \mid x^{u}\right.$ a term in $\left.f\right\}$ is the weighted multiplicity of $f$ at 0 . Although $\tilde{\mathbf{C}}^{n+1}$ is in general not smooth, it is $\mathbf{Q}$-factorial. So we may still apply the adjunction formula (in a suitable sense):

$$
K_{X^{\prime}}=\left.\left(K_{\tilde{\mathbf{C}}^{n+1}}+X^{\prime}\right)\right|_{X^{\prime}} \text { and } K_{X}=\left.\left(K_{\mathbf{C}^{n+1}}+X\right)\right|_{X} .
$$

Putting these together, we get the canonical bundle formula for the $\alpha$-blow-up:

$$
K_{X^{\prime}}=\phi^{*} K_{X}+\left.\left(\alpha\left(x_{0} \cdots x_{n}\right)-\alpha(f)-1\right) E\right|_{X^{\prime}} .
$$

In the two cases considered here one sees easily that $\phi: X^{\prime} \rightarrow X$ is a crepant blow-up.

Write $\alpha=\left(d_{0}, \ldots, d_{n}\right)$. In terms of $\alpha$-weighted homogeneous coordinates, $E=$ $\mathbf{P}\left(d_{0}, \ldots, d_{n}\right)=\mathbf{C}^{n+1} / \sim$ with

$$
\left(Y_{0}, \ldots, Y_{n}\right) \sim\left(\lambda^{d_{0}} Y_{0}, \ldots, \lambda^{d_{n}} Y_{n}\right), \quad \lambda \in \mathbf{C}^{*} .
$$

The standard chart $V_{i}:=\left\{Y_{i} \neq 0\right\}$ thus has the form

$$
\left.\left(Y_{i}^{-d_{0} / d_{i}} Y_{0}, Y_{i}^{-d_{1} / d_{i}} Y_{1}, \ldots, 1 \text { ( } i \text {-th place }\right), \cdots, Y_{i}^{-d_{n} / d_{i}} Y_{n}\right) .
$$

with quasi-coordinates $y_{j}=Y_{i}^{-d_{j} / d_{i}} Y_{j}, j \neq i$. Notice that if $d_{i} \neq 1$, then $V_{i}$ is in general singular.

The corresponding chart $U_{i}$ of $\tilde{\mathbf{C}}^{n+1}$ then has quasi-coordinates $\left(y_{0}, \ldots, y_{n}\right)$ with $y_{i}=x_{i}, y_{j}=Y_{i}^{-d_{j} / d_{i}} Y_{j}, j \neq i$. From (4.5), $\tilde{\mathbf{C}}^{n+1} \subset \mathbf{C}^{n+1} \times \mathbf{P}\left(d_{0}, \ldots, d_{n}\right)$ can 
be described as the set $(x, Y)$ with $x_{i}^{d_{j}} Y_{j}^{d_{i}}=x_{j}^{d_{i}} Y_{i}^{d_{j}}$, and we obtain the mapping $\phi: \tilde{\mathbf{C}}^{n+1} \rightarrow \mathbf{C}^{n+1}$ on $U_{i}$ in terms of coordinates:

$$
\left\{\begin{array}{l}
x_{i}=y_{i}, \\
x_{j}=y_{i}^{d_{j} / d_{i}} y_{j}, \quad j \neq i .
\end{array}\right.
$$

Now let $\tilde{\mathbf{C}}^{4}=\bigcup_{i=0}^{3} U_{i}$ and $f=x_{0}^{2}+x_{1}^{3}+x_{2}^{6}+x_{3}^{k}$ with $k \geq 6$.

Claim 7. $X^{\prime}$ is nonsingular on $U_{i}$ for $i \neq 3$. On $U_{3} \cong \mathbf{C}^{4}, X^{\prime}$ is a hypersurface with equation $f=y_{0}^{2}+y_{1}^{3}+y_{2}^{6}+y_{3}^{k-6}$.

In fact, on $U_{0}$ with quasi-coordinates $\left(y_{0}, \ldots, y_{3}\right)$, the morphism $\phi$ is given by $x_{0}=y_{0}, x_{1}=y_{0}^{2 / 3} y_{1}, x_{2}=y_{0}^{1 / 3} y_{2}, x_{3}=y_{0}^{1 / 3} y_{3}$. So

$$
\begin{aligned}
\phi^{*} f(y) & =f(\phi(y))=y_{0}^{2}+y_{0}^{2} y_{1}^{3}+y_{0}^{2} y_{2}^{6}+y_{0}^{k / 3} y_{3}^{k} \\
& =y_{0}^{2}\left(1+y_{1}^{3}+y_{2}^{6}+y_{0}^{k / 3-2} y_{3}^{k}\right)=y_{0}^{2} g(y) .
\end{aligned}
$$

The only singular point on $U_{0}$ is $y=0$, which does not lie in $X^{\prime}$ since $g(0)=$ $1 \neq 0$. Outside 0 we may regard $\tilde{y_{0}}:=y_{0}^{1 / 3}$ as a coordinate (locally), and an easy calculation of $D g$ as in $\S 3$ shows that $X^{\prime}$ is nonsingular on it. Similar calculation shows also that $X^{\prime}$ is nonsingular on $U_{i}$ for $i \neq 3$.

Now consider $U_{3}$ with coordinates $\left(y_{0}, \ldots, y_{3}\right)$. The morphism $\phi$ is given by $x_{0}=y_{3}^{3} y_{0}, x_{1}=y_{3}^{2} y_{1}, x_{2}=y_{3} y_{2}, x_{3}=y_{3}$. Notice that by (4.6) the chart $U_{3}$ is isomorphic to $\mathbf{C}^{4}$. So

$$
\begin{aligned}
\phi^{*} f(y) & =f(\phi(y))=y_{3}^{6} y_{0}^{2}+y_{3}^{6} y_{1}^{3}+y_{3}^{6} y_{2}^{6}+y_{3}^{k} \\
& =y_{3}^{6}\left(y_{0}^{2}+y_{1}^{3}+y_{2}^{6}+y_{3}^{k-6}\right)=y_{3}^{6} g(y) .
\end{aligned}
$$

The claim is thus proved.

For the theorem, if $k \equiv 0,1 \bmod 6$, then after a finite number of crepant blow-ups we arrive at the case $k=0$ or $k=1$. In both cases we get smooth varieties, and hence we get the crepant resolution of $X$. For other cases, after a finite number of crepant blow-ups we arrive at a hypersurface $X^{\prime}$ when $1<k<6$. These are terminal singularities, since $\sum \frac{1}{a_{i}}=\frac{1}{2}+\frac{1}{3}+\frac{1}{6}+\frac{1}{k}>1+\frac{1}{6}$.

By assumption, we have $k=5$ (a $c E_{8}$ singularity), and it is easy to see that there are no positive integer solutions of

$$
\frac{1}{2}+\frac{a}{3}+\frac{b}{6}+\frac{c}{5}=2
$$

By Theorem $\mathrm{B}, X^{\prime}$ is factorial but singular. Hence by Theorem $1.3, X$ has no crepant resolutions.

The proof of the case $f=x_{0}^{2}+x_{1}^{4}+x_{2}^{4}+x_{3}^{k}$ is entirely the same as the above. We simply use weight $(2,1,1,1)$ instead of $(3,2,1,1)$. Then after a single $(2,1,1,1)$ weighted blow-up we find that $X^{\prime}$ is nonsingular on $U_{i}$ for $i \neq 3$. On $U_{3} \cong \mathbf{C}^{4}$, $X^{\prime}$ is a hypersurface with equation $f=y_{0}^{2}+y_{1}^{4}+y_{2}^{4}+y_{3}^{k-4}$. If $k \equiv 0,1 \bmod 4$, then after a finite number of crepant blow-ups we arrive at smooth varieties. For $k \equiv 3 \bmod 4$, after a finite number of crepant blow-ups we arrive at a hypersurface $X^{\prime}$ given by $y_{0}^{2}+y_{1}^{4}+y_{2}^{4}+y_{3}^{3}=0$. These are terminal singularities, since $\sum \frac{1}{a_{i}}=\frac{1}{2}+\frac{1}{4}+\frac{1}{4}+\frac{1}{3}>1+\frac{1}{4}$. (In fact, it is a $c E_{6}$ singularity.) 
Now there are no positive integer solutions of

$$
\frac{1}{2}+\frac{a}{4}+\frac{b}{4}+\frac{c}{3}=2
$$

So the theorem follows as before.

\section{Q.E.D.}

\section{REFERENCES}

[Fl] H. Flenner, Divisorenklassengruppen quasihomogener Singularitäten, J. Reine Angew. 328 (1981), 128-160. MR 83a:13009

[Ka] Y. Kawamata, The Crepant Blowing-Up of 3-Dimensional Canonical Singularities and Its Applications to Degeneration of Surfaces, Annals of Math. 127 (1988), 93-163. MR 89d:14023

$[\mathrm{KaW}] \mathrm{V}$. Kac and K. Watanabe, Finite linear groups whose ring of invariants is a complete intersection, Bull. AMS 6 (1982), 221-223. MR 83h:14042

[KMM] Y. Kawamata, K. Matsuda, and K. Matsuki Introduction to the minimal model problem, Algebraic Geometry, Sendai, 1985 (T. Oda, ed.). Adv. Stud. Pure Math. vol 10. NorthHolland, Amsterdam, 1987, 283-360. MR 89e:14015

[Ko] J. Kollár, Flops, Nagoya Math. J. 113 (1989), 15-36. MR 90e:14011

[Mi] J. Milnor, Singular Points of Complex Hypersurfaces, Annals of Math. Study 61, Princeton Univ. Press (1968). MR 39:969

[Re1] M. Reid, Canonical threefolds, Journées de Géométrie d'Angers 1979, ed. A. Beauville, Sijthoff \& Noordhoff, Alphen aan den Rijn, 1980, 273-310. MR 82i:14025

[Re2] M. Reid, Minimal models of canonical threefolds, Algebraic Varieties and Analytic Varieties, Tokyo, 1981, Adv. Stud. Pure Math. Vol. 1, ed. S. Iitaka, 131-180 (1983). MR 86a:14010

[Re3] M. Reid, Young person's guide to canonical singularities, Algebraic Geometry Bowdowin 1985, Proc. Symp. Pure Math. 46 (1987), 345-414. MR 89b:14016

[Ro] S.-S. Roan, Minimal Resolution of Gorenstein Orbifolds in Dimension Three, Topology 35 (1996), 489-508. MR 97c:14013

[Sa] P. Samuel, Lectures on Unique Factorization Domains, Tata Instutite of Fundamental Research, Bombay 1964. MR 35:5428

[Sc] M. Schlessinger, Rigidity of Quotient Singularities, Invent. Math. 14 (1971), 17-26. MR 45:5428

National Center for Theoretical Sciences, Mathematics Division, No. 101, Sec. 2, Kuang Fu Road, Hsinchu 30034, Taiwan

Current address: Department of Mathematics, National Central University, Chung-Li (320), Taoyuan, Taiwan

E-mail address: linhw@math.ncu.edu.tw 